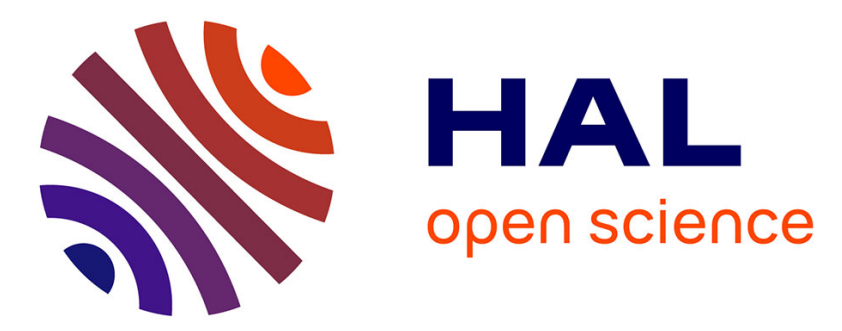

\title{
Vectorial magnetometry using magnetooptic Kerr effect including first- and second-order contributions for thin ferromagnetic films
}

T Kuschel, H Bardenhagen, H Wilkens, R Schubert, J Hamrle, J Pištora, J Wollschläger

\section{To cite this version:}

T Kuschel, H Bardenhagen, H Wilkens, R Schubert, J Hamrle, et al.. Vectorial magnetometry using magnetooptic Kerr effect including first- and second-order contributions for thin ferromagnetic films. Journal of Physics D: Applied Physics, 2011, 44 (26), pp.265003. 10.1088/0022-3727/44/26/265003 . hal-00629466

\section{HAL Id: hal-00629466 https://hal.science/hal-00629466}

Submitted on 6 Oct 2011

HAL is a multi-disciplinary open access archive for the deposit and dissemination of scientific research documents, whether they are published or not. The documents may come from teaching and research institutions in France or abroad, or from public or private research centers.
L'archive ouverte pluridisciplinaire HAL, est destinée au dépôt et à la diffusion de documents scientifiques de niveau recherche, publiés ou non, émanant des établissements d'enseignement et de recherche français ou étrangers, des laboratoires publics ou privés. 


\title{
Vectorial magnetometry using magnetooptic Kerr effect including first and second order contributions for thin ferromagnetic films
}

\author{
T. Kuschel ${ }^{1}$, H. Bardenhagen ${ }^{2}$, H. Wilkens ${ }^{1}$, R. Schubert ${ }^{1}$, \\ J. Hamrle ${ }^{3}$, J. Pištora ${ }^{3}$, and J. Wollschläger ${ }^{1}$ \\ 1 Fachbereich Physik, Universität Osnabrück, \\ Barbarastraße 7, D-49069 Osnabrück, Germany \\ 2 Physikalisches Institut, Universität Würzburg, \\ Am Hubland, D-97074 Würzburg, Germany \\ 3 Department of Physics and Nanotechnology Centre, \\ VSB - Technical University of Ostrava, \\ 17. listopadu 15, 70833 Ostrava-Poruba, Czech Republic
}

(Dated: May 20, 2011)

\begin{abstract}
A new combination of different vectorial magnetometry techniques using magnetooptic Kerr effect is described. The processing of the experimental data contains the separation of linear and quadratic parts of the magnetization curves and determination of all three components of the magnetization vector in units of Kerr rotation without any normalization to the saturation values. The experimental procedure includes measurements with parallel and perpendicular polarized incident light and an external magnetic field parallel and perpendicular to the plane of incidence of light. The determination of the complex Kerr amplitude and the theoretic description of the data processing in assumption of small angles of incidence and also for larger angles of incidence using adequate scaling to the mean saturation value validate this vectorial magnetometry method. In case of an absent out-of-plane component of the magnetization vector, the complete reversal process can easily be reconstructed and interpreted by mono domain states and domain splitting. The measurement procedure and the processing of the data is demonstrated for an ultra thin epitaxial Fe film on $\mathrm{MgO}(001)$.
\end{abstract}




\section{INTRODUCTION}

The magnetooptic Kerr effect (MOKE) discovered by John Kerr ${ }^{1}$ and reported in 1877 is a powerful tool for analysis of magnetic properties of ferromagnetic samples. Incident linear polarized light is reflected from a surface of a magnetized sample. The reflected light is elliptically polarized and its main axis is rotated with respect to the incident polarization. The angle of rotation and the amount of ellipticity depend on the magnetization of the sample. Thus, after analyzing the reflected light, sample properties like magnetic anisotropy, magnetic domains and the magnetization reversal process can be determined. The first MOKE investigations on thin magnetic films were reported in 1985 by Moog and Bader ${ }^{2}$. Henceforward, MOKE has become an important analysis tool for investigations on thin magnetic films.

One interesting application for MOKE is vectorial magnetometry, abbreviated vector MOKE. The different components of the magnetization vector can be separately determined by this MOKE technique. There have been several possibilities used to realize this. Either the alignment of the external magnetic field was kept constant and the adjustment of the optical components had to be changed ${ }^{3,4}$ or the optical components were not modified and the direction of the external magnetic field had to be varied ${ }^{5,6}$. In this work both methods are combined to have some advantages described below. For a review of the methods used so far some vectorial magnetometry experiments are shortly described.

Florczak and Dahlberg reported on measurements of the in-plane magnetization components with longitudinal MOKE (LMOKE) and transversal MOKE (TMOKE) ${ }^{3}$. LMOKE is generated by the in-plane component of the magnetization parallel to the plane of incidence of light and TMOKE is caused by the in-plane component of the magnetization perpendicular to the plane of incidence of light. The last magnetization component (out-of-plane) generates polar MOKE (PMOKE). Both LMOKE and PMOKE cause a change of polarization of the incident light, while TMOKE changes the intensity of incident parallel polarized light, but no change of polarization.

Usually, the magnetization in thin films is energetically preferred to be in-plane due to the magnetic shape anisotropy. Therefore, an additional signal of the PMOKE can often be neglected. Although the determined Kerr signal for each magnetization component is proportional to its generating magnetization component, the proportionality factors between 
Kerr signals and magnetization components are different. Therefore, the two Kerr signals of the in-plane components (change of polarization for LMOKE, change of intensity for TMOKE) can only be compared qualitatively, but not quantitatively. For example, an exact in-plane magnetization vector cannot directly be reconstructed from the measured data.

A possible solution to determine the relation between the separate components of the magnetization vector is to normalize the Kerr signals to their saturation values as reported by Vavassori ${ }^{7}$. Therefore, either an external magnetic field in the direction of each measured component is needed to saturate the magnetization signal or the saturation value has to be estimated by other measurements and calculations. Vavassori combined both methods: The external magnetic field is applied for both in-plane components and the calculation is done for the out-of-plane component to reconstruct the complete magnetization vector in dependence of the external magnetic field ${ }^{7}$. Although this technique is very successful to determine the magnetization for one specific sample, it has the drawback that different samples can only be compared qualitatively due to the normalization to the saturation magnetization.

Another method to obtain the in-plane magnetization components is described by Daboo et al. $^{5}$. Both components are determined with LMOKE. For one of them the external magnetic field is parallel to the plane of incidence of light. For the other one the sample and the external magnetic field is rotated by $90^{\circ}$. Thus in the latter case, the magnetization component perpendicular to the external magnetic field generates the LMOKE (change of polarization). So, the signals of both in-plane components are obtained with LMOKE and therefore, they are directly comparable without any normalization. Thus, the complete reversal process of the magnetization can be reconstructed and different samples can be compared quantitatively in units of Kerr rotation, only if the out-of-plane magnetization component can be neglected.

If the latter assumption cannot be applied, the out-of-plane component also contributes to the polarization of the detected light and the experimental data may be misinterpreted. The in-plane and out-of-plane components of magnetization, however, can be separated combining signals from parallel (p) and perpendicular (s) polarized incident light. The sum of the signals delivers the out-of plane component and the difference provides the in-planecomponent parallel to the incidence plane of light ${ }^{7}$. 
In this work, we built up a vector MOKE setup to determine all three components of the magnetization vector without normalization to the saturation values and with quantitative comparability in units of Kerr rotation. Therefore, we use a combination of both vector MOKE techniques described above. The components of the magnetization vector, which cause both the LMOKE and the PMOKE are measured with s- and p-polarized light ${ }^{7}$. The third component is measured by a $90^{\circ}$ rotation of the sample and of the external magnetic field $^{5}$. If the out-of-plane component cannot be neglected, the PMOKE is also detected in this case. In order to separate these two components, again measurements with s- and p-polarized light are performed.

In addition, MOKE of the second order in magnetization is detected and included into the analysis. The first theory due to this quadratic MOKE (QMOKE) was proposed in 1965 by Metzger et al. ${ }^{8}$ and the first measured magnetization curve including QMOKE was reported in 1990 by Zhong et al. ${ }^{9,10}$ for Ni-Fe bilayers without the knowledge that it was a quadratic effect in magnetization. Further investigations on QMOKE are reported for Fe films on $\mathrm{MgO}^{11}, \mathrm{Mo} / \mathrm{Al}_{2} \mathrm{O}_{3}{ }^{4}, \mathrm{Au} / \mathrm{Fe} / \mathrm{GaAs}^{12}$ and $\mathrm{Ag} / \mathrm{Au} / \mathrm{GaAs} / \mathrm{Ag}^{13}$. Also the magnetization curves of Co films ${ }^{4,14}$ and Co-based Heusler alloys ${ }^{15,16}$ are influenced by QMOKE.

Since vector MOKE is based on linear MOKE, quadratic contributions to the measured MOKE data have to be separated to determine the magnetization vector from the linear part of the MOKE curves. Exemplary vector MOKE studies on a $7 \mathrm{~nm}$ Fe film deposited on $\mathrm{MgO}(001)$ are reported in this work. The separating of the linear and quadratic part of the magnetization curves is shown and the analysis of the curves including domain splitting and reconstruction of the magnetization vector is demonstrated. A theoretic description of the data processing for cubic and tetragonal crystal structures is added to show that this vectorial magnetometry method is valid even for films with tetragonal distorted unit cells.

\section{EXPERIMENTAL MOKE SETUP AND DATA PROCESSING}

The used MOKE setup pictured in Fig. 1 contains a He-Ne Laser with a wavelength of $\lambda=632.8 \mathrm{~nm}$, a polarizer at $0 / 90^{\circ}(\mathrm{p} / \mathrm{s}$-polarized) and a photoelastic modulator $(\mathrm{PEM})$ with a modulation frequency of $f=50 \mathrm{kHz}$. After converting the polarization signal into an intensity signal by an analyzer (polarizer at $45^{\circ}$ ), the signal was detected by a photo diode. The measured intensity of the $2 f$-signal (sensitive to the Kerr rotation) was obtained using 
the lock-in technique with the modulation frequency as reference signal. The magnetization curves were measured by varying the external magnetic field $\mu_{0} H$ between $-130 \mathrm{mT}$ and $+130 \mathrm{mT}$ with bigger intervals for higher magnetic field values close to the saturation magnetization and smaller intervals for a lower magnetic field to detect the reversal process of the magnetization in more detail. Thus, the characteristic steps during the reversal process of the hysteresis can be better identified. The applied magnetic field was determined by a Hall probe.

The measured intensity signal was calibrated to the Kerr angle by small simultaneous rotation of modulator and analyzer. The rotation angle of the optic components is similar to the rotation angle of the polarization of the reflected light induced by the Kerr effect. Thus, this angle can be related to the change of intensity. The external magnetic field is kept constant during calibration to saturate the magnetization.

Two coordinate systems are defined in Fig. 1. The laboratory frame of reference is denoted $x_{1}, x_{2}, x_{3}$ with $x_{1}$ and $x_{2}$ in-plane aligned perpendicular and parallel to the plane of incidence of light, respectively. The $x_{3}$-axis is out-of-plane and parallel to the plane of incidence of light pointing into the sample. The components of the magnetization vector can here be described by $M_{1}, M_{2}$, and $M_{3}$. The crystal frame of reference is defined as $x_{1}^{\prime}$, $x_{2}^{\prime}, x_{3}^{\prime}$ with $x_{1}^{\prime}=\mathrm{Fe}[100], x_{2}^{\prime}=\mathrm{Fe}[010]$ and $x_{3}=x_{3}^{\prime}=\mathrm{Fe}[001]$ of the Fe crystal lattice. Here, the magnetization vector is described by $M_{1}^{\prime}, M_{2}^{\prime}$, and $M_{3}^{\prime}$. This coordinate system is used in the Appendix A to present the validity of the processing. The azimuthal sample angle $\alpha$ is the angle between $x_{2}$ and $x_{2}^{\prime}$ (rotation angle of the sample with respect to the plane of incidence of light). The positive s-direction of the linear polarized light is aligned in the $x_{1}$-direction. The positive p-direction is defined so that $\mathrm{s}, \mathrm{p}$, and direction of propagation of light are right-handed.

In Fig. 2 the measurement procedure and the processing of the data are demonstrated. A theoretical description of this processing is presented in the appendices. The complex amplitude of the Kerr rotation including the second order in magnetization is calculated in Appendix A. In Appendix B it is shown that the processing of the measured data is valid also for non-vanishing out-of-plane magnetization component and second order contributions.

Magnetization hysteresis curves for one complete sample rotation denoted by the azimuthal sample angle $\alpha$ are measured with different orientation of the external magnetic field (parallel and perpendicular to the plane of incidence of light) and with different po- 
larization of the incident light ( $\mathrm{s}$ and $\mathrm{p}$ ). The achieved four data sets are indicated with rectangles in Fig. 2. The further data processing (rhombi) include separation of odd and even parts of the curves to extract the linear and quadratic parts of the magnetic process. The different intermediary results are indicated with ellipses. The different steps are labeled with the numbers of the figures in this article and with the equations in Appendix B.

In order to obtain a sufficient large LMOKE signal, the angle of incidence of $45^{\circ}$ was chosen. For smaller angles of incidence the LMOKE signal decreases and the PMOKE signal increases. Because of the different reflection coefficients for s- and p-polarized light at an angle of incidence of $45^{\circ}$ the measured Kerr effect signals are of slightly different magnitude. Therefore, the linear parts of the hysteresis curves are scaled to the mean saturation value between s- and p-polarized light using the scaling factors $k_{p}$ and $k_{s}$ (cf. Fig. 2). A detailed definition of $k_{p}$ and $k_{s}$ can be found in Appendix B. These factors are determined from the mean saturation value of the curves obtained with external magnetic field parallel to the plane of incidence of light. The difference and the sum of the scaled linear curves result in the components of the magnetization vector $M_{1}, M_{2}$ and $M_{3}$. Thus, the comparability of different samples is verified because the hystereses are not normalized to the saturation values.

In case of $M_{3}=0$ (no out-of-plane component), the magnitude of the magnetization vector and its azimuthal rotation angle with respect to the direction of the positive external magnetic field can easily be determined. Thus, the complete reversal process can be pictured directly in a polar plot of the magnetization vector. The complete calculation of the magnetization vector from the experimental data including the separation of the linear MOKE and the quadratic part of the magnetization curves is performed by an in-house developed analysis software named MOKE Analyzer ${ }^{17}$.

\section{SAMPLE PREPARATION AND STRUCTURAL PROPERTIES}

The Fe film used for exemplary studies was assembled by molecular beam epitaxy (MBE) in a high vacuum chamber at room temperature. Using electron beam bombardment Fe was sublimated from a rod by heating (applied voltage $1 \mathrm{kV}$ ). The Fe deposition rate amounted to $1 \mathrm{~nm} / \mathrm{min}$ at normal incidence. During evaporation the pressure in the preparation chamber was $p=10^{-7}$ mbar (base pressure $10^{-8} \mathrm{mbar}$ ). 
Before film preparation, the $\mathrm{MgO}$ substrate was annealed up to $1000 \mathrm{~K}$ for $12 \mathrm{~h}$ to obtain a clean and well ordered surface. X-ray photoelectron spectroscopy (XPS) was used to check for contamination. After cooling down the substrate with a cooling rate of $5 \mathrm{~K} / \mathrm{min}$ the quality of the $\mathrm{MgO}(001)$ surface structure was tested with low energy electron diffraction (LEED). The quality of the deposited Fe film was also approved by LEED and XPS. Thereafter, the Fe film was capped by amorphous Si to avoid oxidation after transfer to ambient conditions to perform the MOKE studies.

The film thickness and structure were also determined ex-situ by X-ray reflectivity (XRR), X-ray diffraction (XRD) and grazing incidence X-ray diffraction (GIXRD). The measurements were performed at HASYLAB (DESY, Hamburg, Germany) by synchrotron radiation in $\vartheta-2 \vartheta$ scattering geometry at beamline W1 with a photon energy of $10.5 \mathrm{keV}$ $(\lambda=1.18 \mathrm{~nm})$.

These structural techniques confirm the well-known epitaxial properties of Fe on $\mathrm{MgO}(001)^{18}$. Fe grows in the bcc structure and the cubic Fe unit cell is $45^{\circ}$ rotated in-plane to the cubic rock salt unit cell of the $\mathrm{MgO}(001)$ substrate, so Fe[100] \| MgO[110] and $\mathrm{Fe}[001] \| \mathrm{MgO}[001]$. The thickness of the Fe film studied here is $7 \mathrm{~nm}$ and its vertical lattice constant $a_{\perp}$ amounts to $2.83 \AA$, which is $1.4 \%$ less than the bulk value of $2.87 \AA$. This is caused by a tetragonal distortion of the Fe film due to its lateral expansion of $1.0 \%\left(a_{\|}=2.90 \AA\right)$. Although this tetragonal distortion is relatively small, we probed the validity of the presented data processing for cubic and tetragonal crystal structure in the appendices. At the end of Appendix B it is proved that a tetragonal crystal structure can be analyzed the same way as a cubic one. So, the introduced data processing is valid in both cases. Additional structural details of the exemplary $\mathrm{Fe} / \mathrm{MgO}$ sample will be published elsewhere.

\section{VECTOR MOKE RESULTS}

An exemplary measured magnetization curve of the Fe film is shown in Fig. 3. The measurement was performed with s-polarized incident light and external magnetic field parallel to the plane of incidence of light. The curve is separated into an even and an odd part. The even part includes an offset, originating from two sources, the offset coming from the experimental setup and the offset from the contributing QMOKE itself. The offset coming from the experimental setup is generated e.g. by small movement of the laser spot on the 
photo diode after rotating the sample. In principle, it can be separated from the QMOKE offset by sets of measurements, applying an external magnetic saturation field in eight different directions while the sample position is fixed ${ }^{15,19}$. Since only the linear part of the magnetization curve is important for vectorial magnetometry, we concentrate on the odd part of the magnetization curve which equates this linear part in case of no exchange bias effects. The curve represents the typical hysteretic behavior as shown in the inset of Fig. 3 which focuses on small magnetic field strength.

Using Eq. (B3) for the linear part of the magnetization curves for s- and p-polarized light the in-plane components of the magnetization vector can be determined. In case of the external magnetic field parallel to the plane of incidence of light and to a magnetic hard in-plane axis of the sample magnetization curves for s- and p-polarized light are shown in Fig. 4(a). The difference and the sum of both signals with different polarizations result in $M_{2}$ and $M_{3}$ as pictured in Fig. 4(b). Since the out-of-plane component $M_{3}$ does not exist during the entire reversal process, we can describe the complete reversal process using only the in-plane components $M_{1}$ and $M_{2}$.

To check the validity of this calculation with a present out-of-plane component, the external magnetic field is rotated by $28^{\circ}$ with respect to the sample surface to induce a nonvanishing component $M_{3}$. The incident angle is reduced to $0.2^{\circ}$ off the normal of the sample surface to get a larger PMOKE signal and to decrease simultaneously the LMOKE signal. In Fig. 5(a) the magnetization curves for s- and p-polarized light are pictured. Small hysteresis shapes are still present combined with a linear slope due to a part of the external magnetic field in out-of-plane direction. The curve of the resulting $M_{2}$ component (Fig. 5(b)) shows a typical hysteresis, but the amount of Kerr rotation is reduced due to the small incident angle. The $M_{3}$ component does not vanish as it is the case for the set-up with only in-plane external magnetic field (compare Fig. 4(b)). This behavior points to the existence of a forced out-of-plane component $M_{3}$. Going back to the first set-up and the measurements of Fig. 4, thus, we proved that the $M_{3}$ component is negligible.

Magnetization curves of different azimuthal directions $\alpha$ are measured, too. After separating linear and quadratic part $M_{1}$ and $M_{2}$ are obtained from s- and p-polarized signal. In Fig. 6 curves are shown for (a) magnetic easy axis, (b) $5^{\circ}$ off the magnetic hard axis and (c) magnetic hard axis of the sample. Please, note there is still a small difference $\left(<1^{\circ}\right)$ between the measured direction indicated 'magnetic hard axis' and the real magnetic hard 
axis due to small misalignment of the sample.

All curves for $M_{2}$ have the same Kerr rotation for the magnetic saturation of about 72 mdeg, but they differ in the shape of the hysteresis loop. They also vary in the number of magnetization steps for each branch during the reversal process and in the magnitude of the magnetic remanence (magnetization for zero external magnetic field). On the one hand, for the magnetic easy and hard axis only one step in the magnetization curve is observed. On the other hand, for the direction $5^{\circ}$ off the magnetic hard axis two steps are obtained as shown in the inset of Fig. 6(b). The magnetic remanence of the magnetization curves is large in a magnetic easy axis and small in a magnetic hard axis.

Also, the shape of the curves for $M_{1}$ in Fig. 6 varies for the different directions. In magnetic easy direction nearly no $M_{1}$ component is detectable during the entire reversal process as shown in Fig. 6(a). In magnetic hard direction and $5^{\circ}$ off the magnetic hard axis a $M_{1}$ component is obtained, but the shape of theses magnetization curves differs for both crystallographic directions. For example, the coercive field is much smaller for the magnetic hard direction compared to the direction $5^{\circ}$ off the magnetic hard axis. The Kerr rotation for the magnetic saturation vanishes for the magnetic easy as well as hard axis, but for the direction $5^{\circ}$ off the magnetic hard axis a Kerr rotation is still present. Effects like unsaturated magnetization vector or linear background from optical components due to stray field is ruled out as the loops in Fig. 6(a) and (c) do not have this offset.

Magnetization hystereses for many different crystallographic directions (azimuthal sample angle $\alpha$ ) have been recorded to obtain more detailed information. In Fig. 7 the magnetic remanence of several in-plane directions for the $M_{1}$ component (circles) and the $M_{2}$ component (squares) is plotted against the azimuthal sample angle $\alpha$. The observed fourfold magnetic anisotropy originates in the cubic crystal structure of the Fe bcc lattice ${ }^{20}$. Combining both curves in Fig. 7 one can detect four nearly perfect circles. This nice indication of the fourfold magnetic structure is due to sine and cosine characteristic for the projection of $\vec{M}$ in magnetic remanence to the direction perpendicular and parallel to the plane of incidence of light, respectively. The directions with low magnetic remanence for $M_{1}$ and large magnetic remanence for $M_{2}$ are due to the magnetic easy axes, which equate to the [100], [010], [100], and [010] directions of the Fe bcc lattice. The magnetic hard axes (low magnetic remanence for $M_{2}$ and large magnetic remanence for $M_{1}$ ) equate to the [110], [110], $[\overline{1} \overline{1} 0]$, and $[1 \overline{1} 0]$ directions, respectively. 
For the reconstruction of the reversal process the magnitude $|\vec{M}|=\sqrt{M_{1}^{2}+M_{2}^{2}}$ and the azimuthal rotation angle $\gamma=\arctan \left(M_{1} / M_{2}\right)$ of the in-plane magnetization vector $\vec{M}$ are calculated with respect to the positive external magnetic field, since the out-of-plane $M_{3}$ component is absent due to the magnetic shape anisotropy as proved by our analysis.

In Fig. 8 the dependence of the magnitude $|\vec{M}|$ on the external magnetic field is shown for the three crystallographic directions introduced above. The numbers in the curves denote the sequence of the reversal process. The saturation values of all curves amounts to $72 \mathrm{mdeg}$ (sequences no. 1, 3, 4 and 6 in Fig. 8). This is in agreement to the saturation value of the $M_{2}$ component, because in magnetic saturation the magnetization vector should be parallel to the external magnetic field. Notice, that the magnitude for the direction $5^{\circ}$ off the magnetic hard axis exhibits the same saturation value as in case of the other loops, although in this case the $M_{1}$ component is not saturated.

During the reversal process $|\vec{M}|$ is nearly constant for stronger magnetic fields. For lower magnetic fields, however, sharp decreases are observed (sequences no. 2 and 5 in Fig. 8(a)(c)). For the magnetic easy axis one sharp drop for each branch at $\pm 1.0 \mathrm{mT}$ with nearly vanishing magnetization is obtained (inset in Fig. 8(a)). The curve for the direction $5^{\circ}$ off the magnetic hard axis contains two drops for each branch at $\pm 0.8 \mathrm{mT}$ and $\pm 3.5 \mathrm{mT}$. Here, the minimum magnetization is approximately $1 / \sqrt{2}$ times the saturation value (inset in Fig. 8(b)). In magnetic hard direction one drop for each branch occurs. The critical magnetic fields of $\pm 0.8 \mathrm{mT}$ for these sharp decreases of the magnetization are comparable to the one in magnetic easy direction. The minimal value of the magnetization, however, is higher than for the easy direction (inset in Fig. 8(c)). Additional to the drops slowly decreasing magnetization is observed between $-50 \mathrm{mT}$ and $+50 \mathrm{mT}$ for the magnetic hard axis (sequences no. 1b, 3a, 4b and 6a in Fig. 8(c)). This behavior is related to the transition from magnetic mono domain state into magnetic multi domain state with different magnetization orientations. Therefore, the resulting averaged magnetization vector is smaller in this case.

The azimuthal rotation angle $\gamma$ of the magnetization vector $\vec{M}$, which is defined as the angle between sample magnetization and positive external magnetic field direction, is presented in Fig. 9(a)-(c) during the reversal process for all three crystallographic directions described above. The labeling of the curves is equivalent to the labeling in Fig. 8(a)-(c). By definition, the reversal process starts in saturation at $\gamma=0^{\circ}$ (sequence no. 1 in Fig. 9) with magnetization parallel to the external magnetic field except for the direction $5^{\circ}$ off the 
magnetic hard axis (Fig. 9(b)). Here, the magnetization is initially tilted by $\gamma=9^{\circ}$ with respect to the applied external magnetic field due to the offset in saturation for the $M_{1}$ component in Fig. 6(b).

During the switching of polarity of the external magnetic field the magnetization rotates by $180^{\circ}$ with a steep step for the curves of the magnetic easy and hard axis (sequence no. 2 in Fig. 9(a) and Fig. 9(c)). Decreasing the external magnetic field for the $5^{\circ}$ off direction, first the magnetization slips to the direction of one magnetic easy axis (dashed line for $45^{\circ}$ ). This rotation is followed by two steep $90^{\circ}$ steps to other magnetic easy directions (sequences no. 2a and 2b in Fig. 9(b)), before the magnetization rotates towards the direction of the external magnetic field for large strength of the reversed magnetic field (sequence no. 3 in Fig. 9(b)). Similar shallow steps occur in the curve of the magnetic hard axis (sequence no. 1b, 3a, 4b and 6a in Fig. 9(c)). Switching back the polarity of the external magnetic field, the behavior of the magnetization repeats for all magnetic directions until the magnetization reaches the initial orientation.

Fig. 10 presents a polar plot of the magnetization $|\vec{M}(\gamma)|$, in order to illustrate the complete behavior of the magnetization vector. For comparison to the previous diagrams the numbers of the sequence of the reversal process are included into the polar graphs, too.

The direction of the rotation is clockwise both for the magnetic easy axis and the hard axis following the sequence numbers of the reversal process. For the direction $5^{\circ}$ off the magnetic hard axis the sense of the rotation is counterclockwise except for the parts where the magnetization rotates backwards into the direction of the external magnetic field. These backwards rotating parts are indicated by sequences no. 3 and 6 in Fig. 10(b). If the external magnetic field is not applied exactly in easy or hard axis the magnetization rotates towards the closest magnetic easy axis with respect to the direction of the external magnetic field. Thus, the magnetization in Fig. 10(a) rotates clockwise because the magnetic easy axis is initially slightly below the direction of the external field. In Fig. 10(b) the magnetic easy axis at about $42.5^{\circ}$ is obviously the next one compared with the magnetic easy axis at $-47.5^{\circ}$. Thus, the magnetization rotates counterclockwise. In Fig. 10(c) the external magnetic field is in the direction of the magnetic hard axis and so approximately between two magnetic easy axes. The one at about $-45^{\circ}$ seams to be slightly closer to the direction of the external magnetic field than the one at $45^{\circ}$, so the magnetization turns clockwise. 


\section{DISCUSSION}

The reversal process of the magnetization vector can be interpreted using mono domain states during maximal magnitude of the magnetization vector and domain splitting if the magnitude of the magnetization vector decreases. During domain splitting phase in most cases the magnetic moments of the individual domains are parallel to magnetic easy axes. In Fig. 11, 12 and 13 the interpretation for the three discussed magnetic directions of the Fe film is presented. The labeling of the pictures equates the sequence numbers in Fig. 10. Only the first half of the reversal process is shown, the second half is equivalent due to two-fold symmetry of the process.

In magnetic easy direction a mono domain state exists until the external magnetic field vanishes (sequence no. 1 in Fig. 11). After switching polarity of the external magnetic field and reaching coercive field, two $180^{\circ}$ domain states with antiparallel magnetization exist resulting in a vanishing magnetization vector (sequence no. 2 in Fig. 11). During increase of the reversed external magnetic field, again a mono domain state is generated (sequence no. 3 in Fig. 11).

If the external magnetic saturation field is $5^{\circ}$ off the magnetic hard axis a mono domain state exists initially again. Decreasing the strength of the external magnetic field the magnetization rotates towards the closest easy axis (sequence no. 1 in Fig. 12), which is followed by two $90^{\circ}$ domain states with magnetization perpendicular to each other caused by the negative external magnetic field (sequence no. 2a in Fig. 12). The magnitude of the magnetization vector is in minimum $1 / \sqrt{2}$ times the saturation value, since half of the magnetic moments are aligned in one magnetic easy direction and the other half is oriented parallel to the other closest magnetic easy direction. This magnetization jump is characterized by a straight line in the polar plot due to simultaneous decrease of magnetic moments in one magnetic easy direction and increase of magnetic moments in the other magnetic easy direction. Here, the magnetic domains flip sequentially from the first magnetic easy direction into the second. Thus, the resulting magnetization vector follows a straight line and cannot be smaller than $1 / \sqrt{2}$ of the saturation value.

Small increase of the external magnetic field generates a second domain splitting into two $90^{\circ}$ domain states. But in this case the magnetic moments of the domains point to different easy axes as it is the case for the first domain splitting. The result is the second jump of 
the magnetization vector (sequence no. $2 \mathrm{~b}$ in Fig. 12). Here again, the characteristic of the magnetization jump is a straight line in the polar plot due to sequential domain flip. After further increase of the external magnetic field, mono domain state is again formed (sequence no. 3 in Fig. 12).

This reversal process is comparable to that one described qualitatively in the literature, e.g. Daboo et al. ${ }^{21,22}$ studied one- and two-jump reversal processes and their occurrence in dependence of azimuthal sample angle and strength of uniaxial anisotropy contributions for Fe on $\mathrm{GaAs}(001)$. In Ref. ${ }^{23}$ a similar reversal process is shown for FeNi films on glass. However, in contrast to our measurements there is no straight line characteristic in the polar plot observed during the switch of the magnetization. This is due to coherent rotation of the magnetization vector instead of domain splitting. The investigated FeNi films, however, have a two-fold uniaxial magnetic anisotropy compared to the four-fold magnetocrystalline anisotropy for $\mathrm{Fe}$ on $\mathrm{MgO}$ studied here.

In magnetic hard direction the mono domain state is only existing at the start of the reversal process applying the external magnetic saturation field (sequence no. 1a in Fig. 13). Domain splitting already occurs, if the external magnetic field is slightly decreased, so that in magnetic remanence the magnetization vector is composed by magnetic moments parallel to two different magnetic easy axes (sequence no. 1b in Fig. 13).

Applying negative external magnetic field the magnetic moments of most domains jump clockwise $90^{\circ}$ further into the next magnetic easy direction. This jump is again characterized by a straight line in the polar plot comparable to the case for the direction $5^{\circ}$ off the magnetic hard axis. But now there are two simultaneous switching processes. One of them is the flip of a bigger amount of magnetic domains clockwise from the $315^{\circ}$ magnetic easy axis to the $225^{\circ}$ axis, which is schematically shown in the lower part of the polar plot. The second flip is performed by a smaller amount of magnetic domains counterclockwise from the third magnetic easy axis $\left(45^{\circ}\right)$ to the fourth $\left(135^{\circ}\right)$. This is shown in the upper part of the polar plot. Summing up the magnetic moments of all domains during this double switch the resulting minimum of $|\vec{M}|$ is smaller than $1 / \sqrt{2}$ of the saturation value. Here, domains exist in all magnetic easy directions with four $90^{\circ}$ domain states (sequence no. 2 in Fig. 13).

Increasing the external magnetic field, only two $90^{\circ}$ domain states remain (sequence no. $3 \mathrm{a}$ in Fig. 13) and with maximal external magnetic field the mono domain state is reached again (sequence no. 3b in Fig. 13). 
Compared to the vectorial magnetometry techniques described in the introduction, the presented technique has some advantages. Since Florczak and Dahlberg ${ }^{3}$ measured LMOKE and TMOKE signal, they were not able to set the obtained magnetization components in relation due to different characteristics of the measured signals (change of polarization for LMOKE, change of intensity for TMOKE). So the reversal process could only be described qualitatively. Furthermore, the measurement is restricted to in-plane magnetization. Daboo et al. ${ }^{5}$ used LMOKE for measuring both in-plane magnetization components by rotating both sample and external magnetic field by $90^{\circ}$. Thus, an in-plane reversal process can completely be described, but again the method is restricted to in-plane magnetization, while the technique presented here is also sensitive to the out-of-plane magnetization.

Using different polarizations ( $\mathrm{s}$ and $\mathrm{p}$ ) for the incident light the in-plane and out-of-plane magnetization component can be separated like Vavassori ${ }^{7}$ reported. Combing the different directions of the external field and the different polarizations of the incident light one can separate all three components of the magnetization vector as shown in this article. In most cases in the literature (e.g. report of Vavassori ${ }^{7}$ ) the magnetization curves are normalized to the saturation magnetization. Here, we present a technique to keep the saturation value in units of Kerr rotation by scaling the curves to the mean saturation magnetization of s- and p-polarized signal. Thus, the comparability persists for different samples. Furthermore, this scaling makes it possible to use larger angles of incidence and the processing is still valid as shown in the appendices. Even if there are quadratic contributions of the Kerr effect or tetragonal distortion in the investigated film, the presented method is able to separate the components of the magnetization vector.

\section{CONCLUSION}

In this work a combined vectorial magnetometry technique is presented, which reveals the complete reversal process of the magnetization vector in units of Kerr rotation. The method includes separation of linear and quadratic contributions, as well as determination of all three magnetization components. For this purpose s- and p-polarized incident light and an external magnetic field parallel and perpendicular to the plane of incidence of light are used. The curves for s- and p-polarized light are scaled to the mean saturation value in order to separate the in-plane and out-of-plane components of the magnetization vector, 
even for larger angles of incidence. In comparison to previous vectorial magnetometry techniques the magnetization curves are not normalized to the saturation value. Thus, different samples can be compared quantitatively in units of Kerr rotation. The reversal processes are advantageously visualized as polar plots of $\vec{M}$. For an exemplary thin Fe film of $7 \mathrm{~nm}$ thickness and three typical crystallographic directions the reversal process is reconstructed.

Multi domain states can easily be identified as parts of the reversal process with reduced magnitude of the magnetization vector. While in magnetic easy direction only $180^{\circ}$ domain states occur, in magnetic hard direction and $5^{\circ}$ off the magnetic hard axis different $90^{\circ}$ domain states exist. Furthermore, in magnetic hard direction domain splitting proceeds before the magnetic remanence is reached. The flip process of the magnetization vector has a straight line characteristic in the polar plot due to sequential flipping of domains from one magnetic easy axis to the next one. In case of the magnetic hard direction two simultaneous switching processes occur for different easy axes. This results again in a straight line characteristic for the magnetization vector, but the minimum magnitude is reduced compared to the $5^{\circ}$ off direction.

Therefore, this careful analysis can be used to obtain the reversal process even for more complex film structures including a present out-of-plane component of the magnetization vector. Since the proportionality factor in-plane (between complex Kerr amplitude and in-plane component of magnetization vector) and the proportionality factor out-of-plane (between complex Kerr amplitude and out-of-plane component of magnetization vector) are not equal, the reversal process can only be reconstructed in units of Kerr rotation. Up to now, the method is restricted to cubic and tetragonal crystal structures and exchange bias effects have to be excluded. Based on the present study, however, experimental methods for analysis of different crystal structures can be investigated.

\section{ACKNOWLEDGMENTS}

Portions of this research were carried out at the light source DORIS III at DESY. DESY is a member of the Helmholtz Association (HGF). We would like to thank Wolfgang Caliebe for assistance in using beamline W1.

Ostrava group acknowledges financial support from CZ.1.05/2.1.00/01.0040 and PIEFGA-2009-254511. 


\section{Appendix A: Complex Kerr amplitude including second order effects for cubic and} tetragonal crystal structure

In the following the magnetooptic Kerr effect is calculated using the permittivity tensor up to second order in magnetization. Furthermore, the complete magnetization vector $\vec{M}$ is considered including its out-of-plane component.

The magnetooptic Kerr effect for s- and p-polarized incident light can be described by a complex Kerr amplitude $\Phi_{s / p}$ which consists of the Kerr rotation $\Theta_{s / p}$ and the Kerr ellipticity $e_{s / p}$. This complex Kerr amplitude $\Phi_{s / p}=\Theta_{s / p}-i e_{s / p}$ depends on the complex reflection coefficients $\left(r_{s s}, r_{s p}, r_{p p}, r_{p s}\right)$ and therefore on the components of the permittivity tensor $\epsilon_{i j}$. For magnetic thin films the complex Kerr amplitude for s- and p-polarized incident light is ${ }^{15}$

$$
\begin{aligned}
& \Phi_{s}=-\frac{r_{p s}}{r_{s s}}=A_{s}\left(\epsilon_{21}-\frac{\epsilon_{23} \epsilon_{31}}{\epsilon_{d}}\right)+B_{s} \epsilon_{31}, \\
& \Phi_{p}=\frac{r_{s p}}{r_{p p}}=-A_{p}\left(\epsilon_{12}-\frac{\epsilon_{32} \epsilon_{13}}{\epsilon_{d}}\right)+B_{p} \epsilon_{13} .
\end{aligned}
$$

Although this relation was originally derived for film thickness $t \ll \lambda /(4 \pi N)$ with the wavelength $\lambda$ of the incident light and the refractive index $N$ of the magnetic film, it is also valid for a general film thickness ${ }^{24}$. The permittivity of zeroth order of the magnetic film is $\epsilon_{d}$ and the complex weighting coefficients $A_{s / p}$ and $B_{s / p}$ depend on the angle of incidence of light and on the optical surroundings (overlayers, underlayers) of the magnetic film.

The components of the permittivity tensor $\epsilon_{i j}$ depend on the magnetization vector $\vec{M}=$ $\left(M_{1}, M_{2}, M_{3}\right)$. The exact dependence is related to the crystal structure of the magnetic material in the film. The Kerr amplitude $\Phi_{s / p}$ was calculated in Ref. ${ }^{15}$ for cubic crystal structure, in-plane rotation of the sample and the assumption $M_{3}=0$. Here, we calculate $\Phi_{s / p}$ without the assumption of $M_{3}=0$ to show that our data processing can be performed for each possible orientation of $\vec{M}$ including second order effects. Furthermore, we study the Kerr effect of tetragonal crystal structure to include films with tetragonal distorted unit cells. Afterwards, the results are compared with the calculations for cubic crystal structure.

We use the cartesian coordinate system $x_{1}, x_{2}, x_{3}$ pictured in Fig. 1 which is the laboratory frame of reference. The $x_{1}$ - and $x_{2}$-axes are in-plane aligned with respect to the sample surface and the $x_{3}$-axis corresponds to the surface normal (out-of-plane) pointing into the film. The $x_{1}$-direction is perpendicular and the $x_{2}$-direction parallel to the plane of incidence of light. The components $M_{1}, M_{2}$ and $M_{3}$ are denoted with respect to the $x_{1^{-}}, x_{2^{-}}$and $x_{3^{-}}$ 
directions, respectively. Furthermore, a second coordinate system is defined as the crystal frame of reference which is independent of the position of the sample in the setup. Here, the $\mathrm{Fe}[100], \mathrm{Fe}[010]$ and $\mathrm{Fe}[001]$ direction of the crystal are parallel to the $x_{1^{-}}^{\prime}, x_{2}^{\prime}$ and $x_{3}^{\prime}$-axis of the cartesian coordinate system of the crystal frame of reference, respectively. The $x_{3}^{\prime}$-axis is parallel to the $x_{3}$-axis.

In the laboratory frame of reference each element $\epsilon_{i j}$ of the permittivity tensor $\hat{\epsilon}$ can be expressed as

$$
\epsilon_{i j}=\epsilon_{i j}^{(0)}+K_{i j k} M_{k}+G_{i j k l} M_{k} M_{l}+\cdots
$$

using the constant permittivity term of zeroth order $\epsilon_{i j}^{(0)}$, the elements $K_{i j k}$ of the linear magnetooptic tensor $\hat{K}$, the elements $G_{i j k l}$ of the quadratic magnetooptic tensor $\hat{G}$ and the Einstein summation convention. Furthermore, some general symmetry arguments are valid which $\operatorname{are}^{25}$

$$
\begin{aligned}
\epsilon_{i j}^{(0)} & =\epsilon_{j i}^{(0)}, \\
K_{i j k} & =-K_{j i k}, \quad K_{i i k}=0, \quad i \neq j \neq k, \\
G_{i j k l} & =G_{j i k l}=G_{j i l k}=G_{i j l k} .
\end{aligned}
$$

The expressions concerning the crystal frame of reference are now defined as $\epsilon_{i j}^{(0)^{\prime}}, K_{i j k}^{\prime}$ and $G_{i j k l}^{\prime}$. Eq. (A2) and (A3) are also fulfilled here. Furthermore, for a tetragonal crystal structure the number of independent tensor elements up to second order in magnetization is reduced to

$$
\begin{aligned}
\epsilon_{11}^{(0)^{\prime}}=\epsilon_{22}^{(0)^{\prime}}:=\epsilon_{d}, \quad \epsilon_{33}^{(0)^{\prime}}:=\epsilon_{d_{33}}, \\
\epsilon_{i j}^{(0)^{\prime}}=0, \quad i \neq j, \\
K_{231}^{\prime}=K_{312}^{\prime}:=K_{31}, \quad K_{123}^{\prime}:=K_{12}, \\
G_{1111}^{\prime}=G_{2222}^{\prime}:=G_{11}, \quad G_{3333}^{\prime}:=G_{33}, \\
G_{1122}^{\prime}=G_{2211}^{\prime}:=G_{12}, \quad G_{1133}^{\prime}=G_{2233}^{\prime}:=G_{13}, \\
G_{3311}^{\prime}=G_{3322}^{\prime}:=G_{31}, \\
G_{2323}^{\prime}=G_{3131}^{\prime}:=G_{44}, \quad G_{1212}^{\prime}:=G_{66} .
\end{aligned}
$$

Consider that the permutation rules of Eq. (A3) are still valid. This yields to a permittivity tensor for tetragonal crystal structure of 
$\hat{\epsilon}=\left(\begin{array}{ccc}G_{11} M_{1}^{\prime 2}+G_{12} M_{2}^{\prime 2}+G_{13} M_{3}^{\prime 2}+\epsilon_{d} & 2 G_{66} M_{1}^{\prime} M_{2}^{\prime}+K_{12} M_{3}^{\prime} & 2 G_{44} M_{3}^{\prime} M_{1}^{\prime}-K_{31} M_{2}^{\prime} \\ 2 G_{66} M_{1}^{\prime} M_{2}^{\prime}-K_{12} M_{3}^{\prime} & G_{12} M_{1}^{\prime 2}+G_{11} M_{2}^{\prime 2}+G_{13} M_{3}^{\prime 2}+\epsilon_{d} & 2 G_{44} M_{2}^{\prime} M_{3}^{\prime}+K_{31} M_{1}^{\prime} \\ 2 G_{44} M_{3}^{\prime} M_{1}^{\prime}+K_{31} M_{2}^{\prime} & 2 G_{44} M_{2}^{\prime} M_{3}^{\prime}-K_{31} M_{1}^{\prime} & G_{31}\left(M_{1}^{\prime 2}+M_{2}^{\prime 2}\right)+G_{33} M_{3}^{\prime 2}+\epsilon_{d_{33}}\end{array}\right)$

where $M_{i}^{\prime}$ denote the components of the magnetization in the crystal frame of reference. For the azimuthal sample angle $\alpha=0$ this matrix is also valid in the laboratory frame of reference. In order to determine the dependence of the elements of the permittivity tensor regarding $\alpha$, the permittivity tensor of the crystal structure has to be transformed using the rotation matrix

$$
\hat{R}=\left(\begin{array}{ccc}
\cos (\alpha) & -\sin (\alpha) & 0 \\
\sin (\alpha) & \cos (\alpha) & 0 \\
0 & 0 & 1
\end{array}\right)
$$

keeping the magnetization vector $\vec{M}$ fixed with respect to the laboratory frame of reference. This is done by rotating the permittivity tensor of zeroth order $\hat{\epsilon}^{(0)}$ and both magnetooptic tensors $\hat{K}$ and $\hat{G}$ independent of $\vec{M}$ using again the Einstein convention and the equations

$$
\begin{aligned}
\epsilon_{i j}^{(0)} & =R_{i m} R_{j n} \epsilon_{m n}^{(0)^{\prime}}, \\
K_{i j k} & =R_{i m} R_{j n} R_{k o} K_{m n o}^{\prime}, \\
G_{i j k l} & =R_{i m} R_{j n} R_{k o} R_{l p} G_{m n o p}^{\prime} .
\end{aligned}
$$

After this rotation $\hat{\epsilon}^{(0)}$ and $\hat{K}$ do not change for tetragonal symmetry. However, the quadratic magnetooptic tensor $\hat{G}$ changes. Only off-diagonal elements of the permittivity tensor are taken into account for determination of the Kerr effect (cf. Eq. (A1)). Thus, we focus on the off-diagonal elements of the rotated permittivity tensor up to second order. The result 
using Eq. (A2) for the rotated tensors yields to

$$
\begin{aligned}
\epsilon_{12 / 21}= & {\left[2 G_{66}+\frac{\Delta G}{2}(1-\cos (4 \alpha))\right] M_{1} M_{2} } \\
& -\frac{\Delta G}{4} \sin (4 \alpha)\left(M_{2}^{2}-M_{1}^{2}\right) \pm K_{12} M_{3}, \\
\epsilon_{23 / 32}= & 2 G_{44} M_{2} M_{3} \pm K_{31} M_{1}, \\
\epsilon_{31 / 13}= & 2 G_{44} M_{3} M_{1} \pm K_{31} M_{2} .
\end{aligned}
$$

Here, the magnetooptic anisotropy parameter $\Delta G=G_{11}-G_{12}-2 G_{66}$ is introduced. These off-diagonal elements are inserted into Eq. (A1) for the complex Kerr amplitude to obtain the complex Kerr amplitude up to second order in magnetization which is

$$
\begin{aligned}
\Phi_{s / p}= & \pm A_{s / p}\left[2 G_{66}+\frac{\Delta G}{2}(1-\cos (4 \alpha))-\frac{K_{31}^{2}}{\epsilon_{d}}\right] M_{1} M_{2} \\
& \mp A_{s / p} \frac{\Delta G}{4} \sin (4 \alpha)\left(M_{2}^{2}-M_{1}^{2}\right)+B_{s / p} G_{44} M_{3} M_{1} \\
& \pm B_{s / p} K_{31} M_{2}-A_{s / p} K_{12} M_{3} .
\end{aligned}
$$

So the Kerr amplitude depends on linear terms (which are proportional to $M_{2}$ and $M_{3}$, corresponding to longitudinal and polar MOKE), and second order terms proportional to $M_{1} M_{2}, M_{2}^{2}-M_{1}^{2}$, and $M_{3} M_{1}$. For cubic crystal structure the number of independent coefficients is reduced using $K_{12}=K_{31}, G_{12}=G_{13}=G_{31}$ and $G_{44}=G_{66}$ in Eq. (A9).

\section{Appendix B: Theoretic description of the experimental processing}

Here, from theoretical point of view, we present the validity of the processing, namely the separation of linear and quadratic part of the curves as well as the determination of the separate components of the magnetization vector.

Following the processing of the experimental data in Fig. 2 the odd and even part of the magnetization curve is separated. For systems without exchange bias it is assumed that the magnetic reversal can be described by $\vec{M}_{\text {inc }}(\vec{H})=-\vec{M}_{\text {dec }}(-\vec{H})$ with the magnetization vector $\vec{M}_{\text {inc }}$ for increasing external magnetic field and $\vec{M}_{\text {dec }}$ for decreasing external magnetic field. Thus, symmetrization and antisymmetrization of the Kerr amplitude (odd and even part) result in the linear and quadratic MOKE using ${ }^{15}$

$$
\Phi^{\text {lin/quad }}=\left[\Phi_{\text {inc }}(\vec{H}) \mp \Phi_{\text {dec }}(-\vec{H})\right] / 2
$$


The Kerr amplitude for increasing and decreasing external magnetic field is denoted by $\Phi_{\text {inc }}$ and $\Phi_{\text {dec }}$, respectively. Symmetrization and antisymmetrization of Eq. (A9) neglecting the terms of higher order mentioned above yields to

$$
\begin{aligned}
\Phi_{s / p}^{\text {quad }}= & \pm A_{s / p}\left[2 G_{66}+\frac{\Delta G}{2}(1-\cos (4 \alpha))-\frac{K_{31}^{2}}{\epsilon_{d}}\right] M_{1} M_{2} \\
& \mp A_{s / p} \frac{\Delta G}{4} \sin (4 \alpha)\left(M_{2}^{2}-M_{1}^{2}\right)+B_{s / p} G_{44} M_{3} M_{1} \\
\Phi_{s / p}^{\text {lin }}= & \pm B_{s / p} K_{31} M_{2}-A_{s / p} K_{12} M_{3} .
\end{aligned}
$$

For small angles of incidence no scaling of the curves is necessary, since the Kerr amplitude for s- and p-polarized light are of the same values. In that case, $A_{s}=A_{p} \equiv A$ and $B_{s}=B_{p} \equiv B$ can be assumed. Thus, $\Phi_{s / p}^{\text {lin }}$ can easily be used to obtain the magnetization curves $\Phi_{+}\left(M_{3}\right)$ and $\Phi_{-}\left(M_{2}\right)$ since they are proportional to the components $M_{3}$ and $M_{2}$ of the magnetization vector, respectively ${ }^{7}$. The result is

$$
\begin{aligned}
& \Phi_{+}=-\frac{\Phi_{s}^{\mathrm{lin}}+\Phi_{p}^{\operatorname{lin}}}{2}=A K_{12} M_{3} \\
& \Phi_{-}=\frac{\Phi_{s}^{\mathrm{lin}}-\Phi_{p}^{\operatorname{lin}}}{2}=B K_{31} M_{2} .
\end{aligned}
$$

In order to determine such curves for larger angles of incidence (as it is the case in this study) the latter assumption is not valid any more. Nevertheless, scaling the magnetization curves $\Phi_{s / p}^{\operatorname{lin}}$ to the mean saturation value $\Phi_{+}\left(M_{3}\right)$ and $\Phi_{-}\left(M_{2}\right)$ are still proportional to $M_{3}$ and $M_{2}$, respectively. Applying the external magnetic saturation field $\Phi_{s / p}^{\text {lin,sat }}= \pm B_{s / p} K_{31} M_{2}$ is valid since it is $M_{3}=0$ in magnetic saturation. Thus, the scaling factor $k_{s / p}$ can be described by

$$
k_{s / p}= \pm \frac{\Phi_{s}^{\mathrm{lin}, \mathrm{sat}}-\Phi_{p}^{\mathrm{lin}, \mathrm{sat}}}{2 \Phi_{s / p}^{\mathrm{lin}, \mathrm{sat}}}=\frac{B_{s}+B_{p}}{2 B_{s / p}}
$$

where $\Phi_{s / p}^{\text {lin,sat }}$ are linear Kerr effects in saturation for s- and p-polarized incident light.

Using again Eq. (B3) for the scaled curves $k_{s / p} \Phi_{s / p}^{\text {lin }}$ we obtain

$$
\begin{aligned}
& \Phi_{+}=-\frac{k_{s} \Phi_{s}^{\text {lin }}+k_{p} \Phi_{p}^{\text {lin }}}{2}=\frac{B_{s}+B_{p}}{4}\left(\frac{A_{s}}{B_{s}}+\frac{A_{p}}{B_{p}}\right) K_{12} M_{3}=\frac{B_{s}+B_{p}}{2} C K_{12} M_{3} \\
& \Phi_{-}=\frac{k_{s} \Phi_{s}^{\text {lin }}-k_{p} \Phi_{p}^{\operatorname{lin}}}{2}=\frac{B_{s}+B_{p}}{4}\left[2 K_{31} M_{2}+\left(\frac{A_{p}}{B_{p}}-\frac{A_{s}}{B_{s}}\right) K_{12} M_{3}\right]=\frac{B_{s}+B_{p}}{2} K_{31} M_{2} .
\end{aligned}
$$


Thus, $\Phi_{+}$is proportional to $M_{3}$ independent of $A_{s / p}$ and $B_{s / p}$, while $\Phi_{-}$is proportional to $M_{2}$ because $A_{s} / B_{s}=A_{p} / B_{p}=C$. This comes directly from the definition of the Kerr effect as the polar component is $\Phi_{s / p}^{\mathrm{pol}}=A_{s / p} K_{12} M_{3}=\mp r_{p s / s p}^{\mathrm{pol}} / r_{s s / p p}$, whereas the longitudinal one writes $\Phi_{s / p}^{\text {lon }}= \pm B_{s / p} K_{31} M_{2}=\mp r_{p s / s p}^{\text {lon }} / r_{s s / p p}$. As follows from symmetry arguments and when s- and p-directions are defined as in Fig. 1, $r_{s p}^{\mathrm{pol}}=r_{p s}^{\mathrm{pol}}$ and $r_{s p}^{\text {lon }}=-r_{p s}^{\text {lon }}$. Hence, $A_{s} / B_{s}=r_{p s}^{\mathrm{pol}} / r_{p s}^{\mathrm{lon}}=C, A_{p} / B_{p}=-r_{s p}^{\mathrm{pol}} / r_{s p}^{\text {lon }}=r_{p s}^{\mathrm{pol}} / r_{p s}^{\text {lon }}=C$. All those assumption are valid independent of the angle of incidence ${ }^{24}$. Thus, there is no dependence on $M_{3}$ for $\Phi_{-}$ in Eq. (B5) any more. Hence, it allows to separate polar and longitudinal direction, using only one scaling, in direction, where it is easier to saturate the sample (in our case here for in-plane magnetization, but it can be easily generalized for scaling in out-of-plane direction).

However, this is valid in case if complex description of $A_{s / p}, B_{s / p}$ etc. is kept for the analysis. Hence, to determine the pure $M_{2}$ signal, one would need to measure the full complex Kerr effect (both Kerr rotation and Kerr ellipticity). However, in our case here, we have measured only Kerr rotation, providing $\Theta_{s / p}^{\mathrm{pol}, \text { lon }}=\Re\left(\Phi_{s / p}^{\mathrm{pol}, \mathrm{lon}}\right)= \pm \Re\left(B_{s / p} K_{31}\right) M_{2}-$ $\Re\left(A_{s / p} K_{12}\right) M_{3}$ with the real part $\Re(z)$ of $z$. As in general $\Re\left(A_{s / p} / B_{s / p}\right) \neq \Re\left(A_{s / p}\right) / \Re\left(B_{s / p}\right)$, the relation used for $\Phi_{-}$in Eq. (B5), $A_{s} / B_{s}=A_{p} / B_{p}$, is valid only approximately. For example, if only Kerr rotation is measured, and if $M_{2}$ is expressed, then for incidence angle of $30^{\circ}$ and $45^{\circ}$, the 'cross talk' of $M_{3}$ to the separated $M_{2}$ is $18 \%$ and $30 \%$, respectively. This example is numerically calculated for the case of our investigated system $\mathrm{Fe}(7 \mathrm{~nm}) / \mathrm{MgO}$, using the light description inside a multilayer structure ${ }^{26}$. Although this cross talk may look large on first sight, one has to keep in mind that the value of the longitudinal Kerr rotation is about $20 \%$ to $30 \%$ of the polar one, respectively, as calculated for this particular case.

For determination of the signal proportional to the third component of the magnetization vector $M_{1}$ the external magnetic field and the sample have to be in-plane rotated $90^{\circ}$ clockwise. Thus, the coordinate system pictured in Fig. 1 also rotates and the $x_{2}$-axis becomes the $x_{1}$-axis and the $x_{1}$-axis becomes the negative $x_{2}$-axis. The whole theoretic description in this appendix is now valid using $M_{1}$ instead of $M_{2}$ and $-M_{2}$ instead of $M_{1}$. For the scaling of the measured curves in this case again the factors $k_{s / p}$ determined for the setup with external magnetic field parallel to the plane of incidence of light are used. The scaling factors $k_{s / p}$ are the same for the two different alignments of the external field because in both cases the same Kerr effects occur (LMOKE for the in-plane components $M_{1}$ and $M_{2}$, PMOKE for the out-of-plane component $M_{3}$ ). In conclusion, all components of the magnetization vector 
can be determined in units of Kerr rotation.

This separation method is also valid for cubic crystal structure. Here, the number of independent coefficients is reduced using $K_{12}=K_{31}$ in Eq. (B5). So, independent of the amount of tetragonal distortion, the separation of the signals proportional to $M_{2}$ and $M_{3}$ is possible (and separation of $M_{1}$ and $M_{3}$ for $90^{\circ}$ rotated magnet and sample). Only the proportionality factor between the complex Kerr amplitude and the components of the magnetization vector changes compared to the case of tetragonal crystal structure. So, all conclusions are valid for tetragonal and for cubic crystal structure.

1 J. Kerr, Phil. Mag. 3, 321 (1877).

2 E.R. Moog, S.D. Bader, Superlattices and Microstructures 1, 543 (1985).

3 J.M. Florczak, E.D. Dahlberg, J. Appl. Phys. 67, 7520 (1990).

4 R.M. Osgood III, B.M. Clemens, R.L. White, Phys. Rev. B 55, 8990 (1997).

5 C. Daboo, J.A.C. Bland, R.J. Hicken, A.J.R. Ives, M.J. Baird, M.J. Walker, Phys. Rev. B 47, $11852(1993)$.

${ }^{6}$ C. Daboo, J.A.C. Bland, R.J. Hicken, A.J.R. Ives, M.J. Baird, M.J. Walker, J. Appl. Phys. 73, $6368(1993)$.

7 P. Vavassori, Appl. Phys. Lett. 77, 1605 (2000).

8 G. Metzger, P. Pluvinage, R. Torguet, Ann. Phys. (Paris) 10, 5 (1965).

9 Q.-M. Zhong, A.S. Arrott, B. Heinrich, Z. Celinski, J. Appl. Phys. 67, 4448 (1990).

10 Q.-M. Zhong, A.S. Arrott, B. Heinrich, Z. Celinski, J. Magn. Magn. Mater. 104, 1837 (1992).

11 K. Postava, H. Jaffres, A. Schuhl, F. Nguyen van Dau, M. Goiran, A.R. Fert, J. Magn. Magn. Mater. 172, 199 (1997).

12 Shi-shen Yan, R. Schreiber, P. Grünberg, R. Schäfer, J. Magn. Magn. Mater. 210, 309 (2000).

13 M. Buchmeier, R. Schreiber, D.E. Bürgler, C.M. Schneider, Phys. Rev. B 79, 064402 (2009).

14 J.A.C. Bland, M.J. Baird, H.T. Leung, A.J.R. Ives, K.D. Mackay, H.P. Hughes, J. Magn. Magn. Mater. 113, 178 (1992).

15 J. Hamrle, S. Blomeier, O. Gaier, B. Hillebrands, H. Schneider, G. Jakob, K. Postava, C. Felser, J. Phys. D: Appl. Phys. 40, 1563 (2007).

16 O. Gaier, J. Hamrle, S.J. Hermsdoerfer, H. Schultheiß, B. Hillebrands, Y. Sakuraba, M. Oogane, 
Y. Ando, J. Appl. Phys. 103, 103910 (2008).

17 R. Schubert, Bachelor Thesis, University of Osnabrück (2009).

18 J.F. Lawler, R. Schad, S. Jordan, H. van Kempen, J. Magn. Magn. Mater. 165, 224 (1997).

19 K. Postava, D. Hrabovský, J. Pištora, A.R. Fert, Š. Višňovský, T. Yamaguchi, J. Appl. Phys. 91, 7293 (2002).

20 J.L. Costa-Krämer, J.L. Menéndez, A. Cebollada, F. Briones, D. García, A. Hernando, J. Magn. Magn. Mater. 210, 341 (2000).

21 C. Daboo, R.J. Hicken, D.E.P. Eley, M. Gester, S.J. Grey, E. Ahmad, A.J.R. Ives, J.A.C. Bland, J. Appl. Phys. 75, 5586 (1994).

22 C. Daboo, R.J. Hicken, E. Gu, M. Gester, S.J. Grey, D.E.P. Eley, E. Ahmad, J.A.C. Bland, R. Ploessl, J.N. Chapman, Phys. Rev. B 51, 15964 (1995).

23 H. Ohldag, N.B. Weber, F.U. Hillebrecht, E. Kisker, J. Appl. Phys. 91, 2228 (2002).

24 J. Hamrle, J. Ferre, J.P. Jamet, V. Repain, G. Baudot, S. Rousset, Phys. Rev. B 67, 155411 (2003).

25 Š. Višňovský, Czech. J. Phys. B 36, 1424 (1986).

26 Š. Višňovský, Czech. J. Phys. B 36, 625 (1986). 


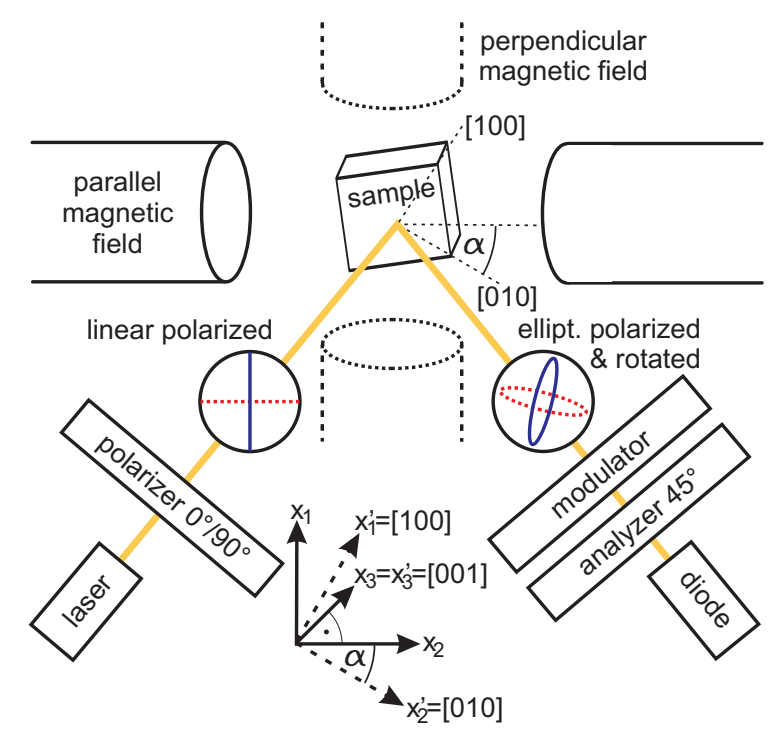

FIG. 1. MOKE setup for the different measurements with s- and p-polarized incident light and external magnetic field parallel and perpendicular to the plane of incidence of light, respectively. Furthermore, the definition of the coordinate systems is shown. The laboratory frame of reference is defined as $x_{1}, x_{2}, x_{3}$ with $x_{1}$ and $x_{2}$ in-plane aligned (perpendicular and parallel to plane of incidence of light, respectively). The crystal frame of reference is defined as $x_{1}^{\prime}, x_{2}^{\prime}, x_{3}^{\prime}$ with $\mathrm{x}_{1}^{\prime}=[100], \mathrm{x}_{2}^{\prime}=[010]$ and $\mathrm{x}_{3}=\mathrm{x}_{3}^{\prime}=[001]$. The azimuthal sample angle $\alpha$ is the angle between $x_{2}$ and $x_{2}^{\prime}$. 


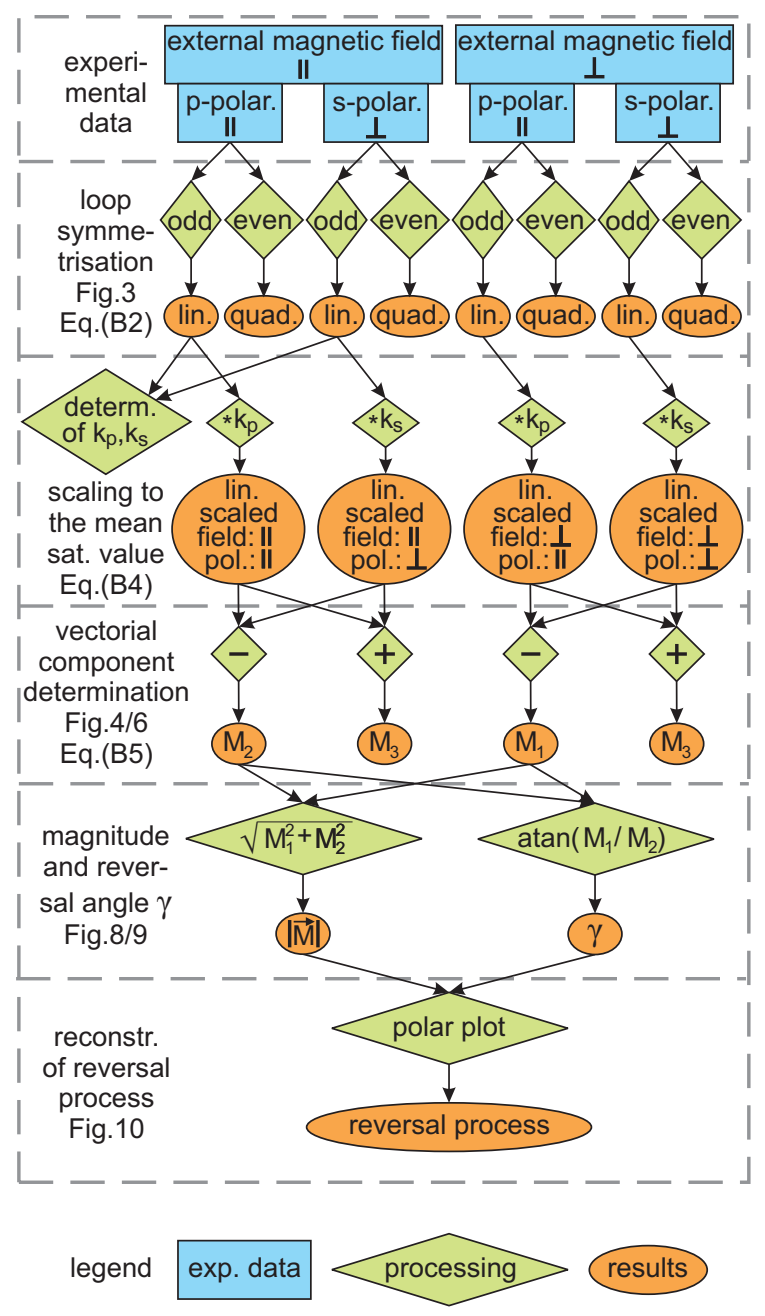

FIG. 2. Schematic drawing of the processing (rhombi) of the experimental data (rectangles). The results (ellipses) are labeled with the numbers of the figures in this article and the equations of the Appendix B. $\|, \perp$ denotes parallel, perpendicular to plane of incidence of light, respectively. The two lower dashed-lined boxes are only valid in case of $M_{3}=0$. 


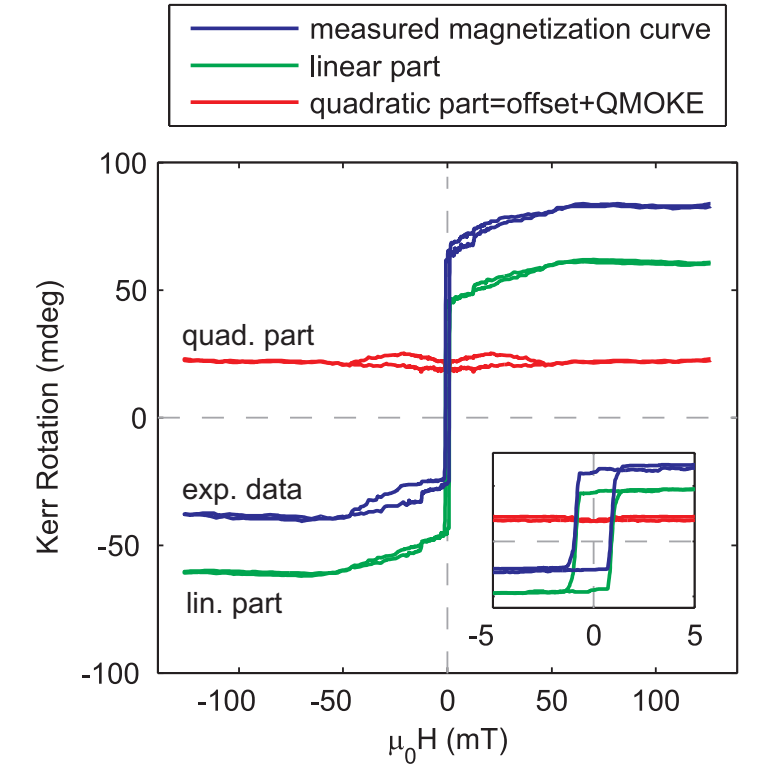

FIG. 3. Separation of the linear and quadratic part of the measured MOKE signal for a magnetization curve in one of the magnetic hard axes. The measurement was performed with s-polarized incident light and an external magnetic field parallel to the plane of incidence of light. The inset is a close-up of the region between $-5 \mathrm{mT}$ and $+5 \mathrm{mT}$. 

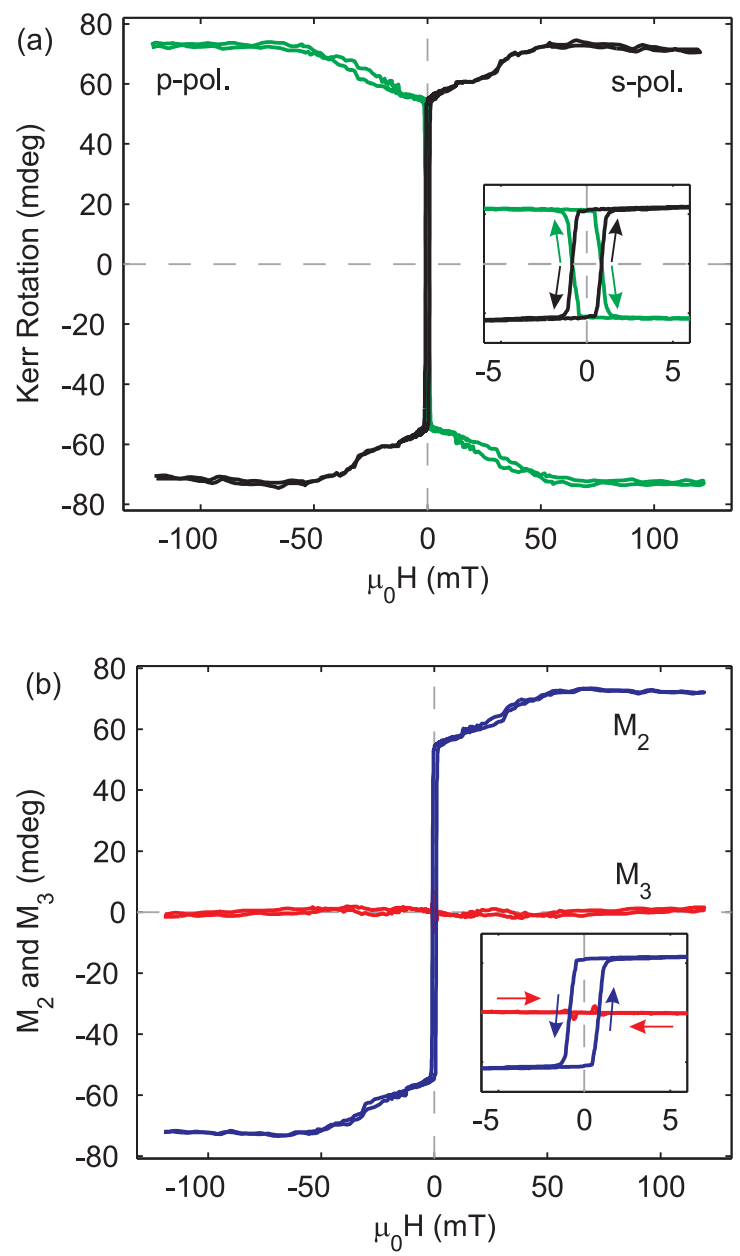

FIG. 4. (a) The linear part of the magnetization curves measured with incident s- and p-polarized light for a magnetic hard axis. (b) Determination of $M_{2}$ and $M_{3}$ from the magnetization curves in (a). The out-of-plane component $M_{3}$ of the magnetization vector does not exist during the reversal process. The insets are close-ups of the regions between $-5 \mathrm{mT}$ and $+5 \mathrm{mT}$. 

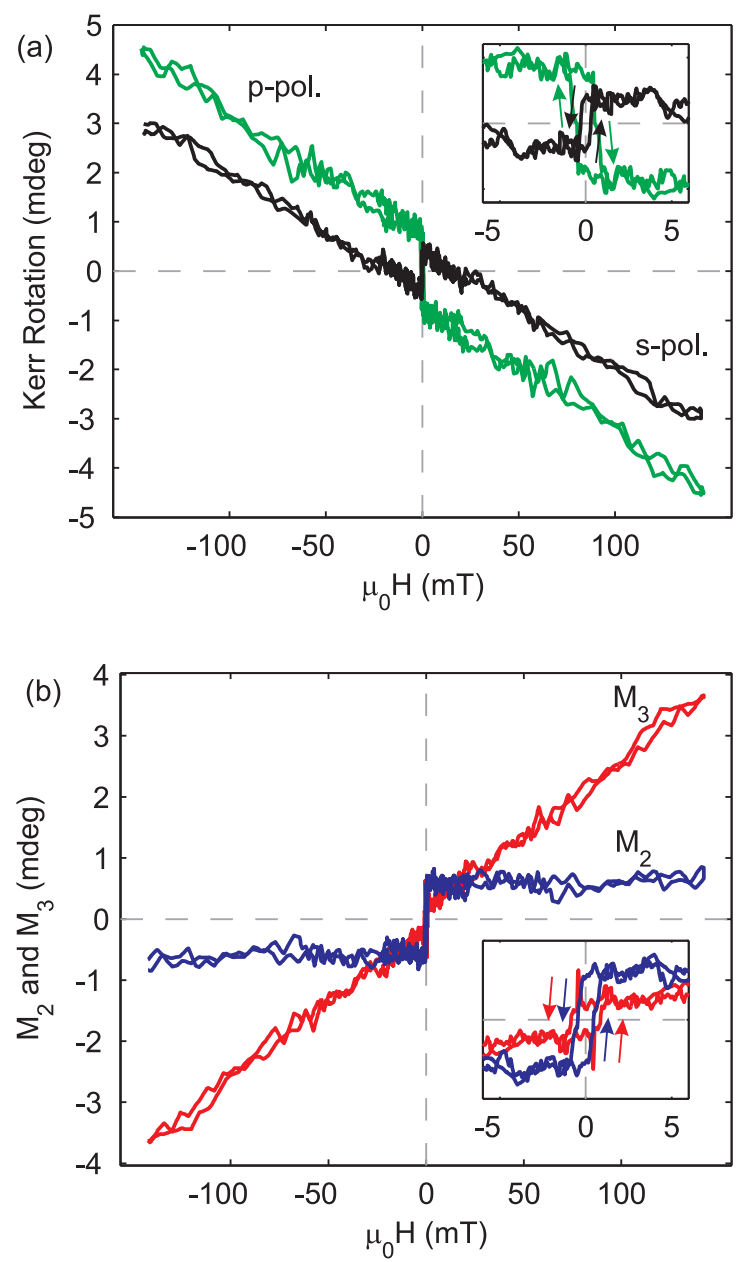

FIG. 5. MOKE measurement with an induced out-of-plane component of the magnetization vector due to a rotated external magnetic field by $28^{\circ}$ with respect to the sample surface and an incident angle of $0.2^{\circ}$ off the normal of the sample surface. (a) The linear part of the magnetization curves measured with incident s- and p-polarized light for a magnetic easy axis. (b) Determination of $M_{2}$ and $M_{3}$ from the magnetization curves in (a). The out-of-plane component $M_{3}$ depends linearly on the external magnetic field. The insets are close-ups of the regions between $-5 \mathrm{mT}$ and $+5 \mathrm{mT}$. 

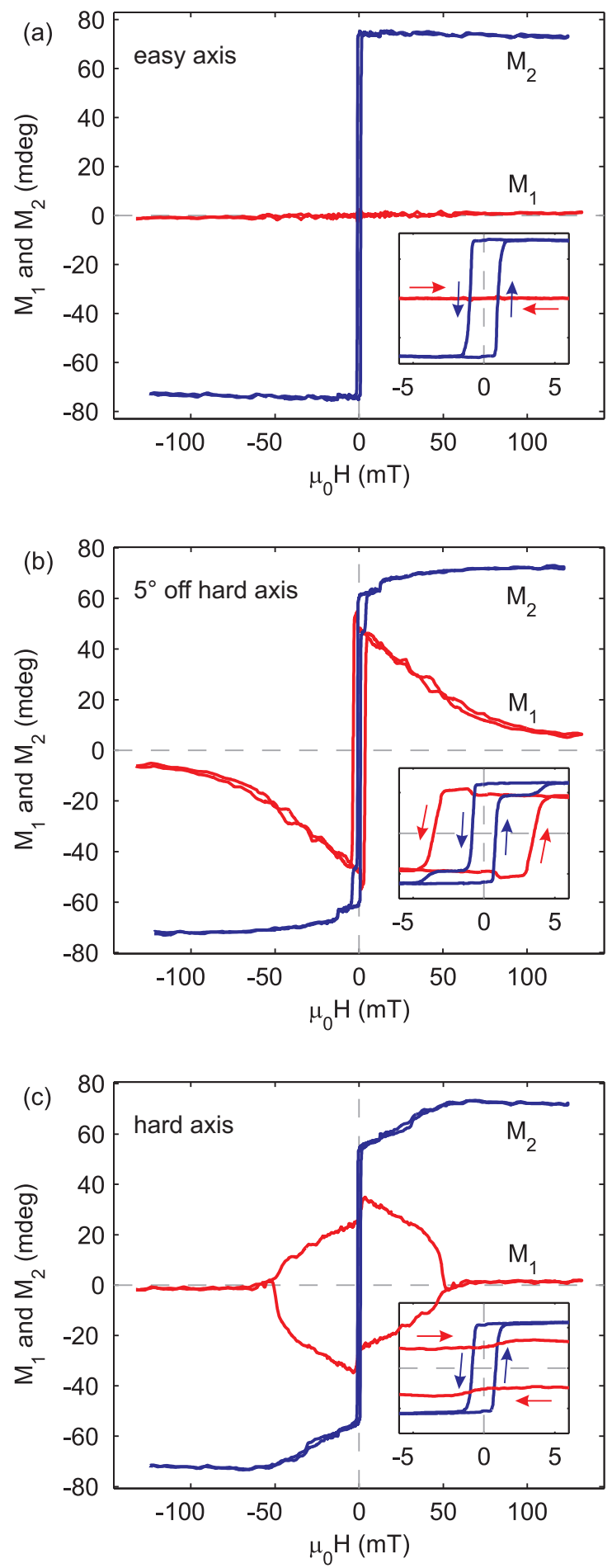

FIG. 6. Magnetization curves for $M_{1}$ and $M_{2}$ for (a) magnetic easy axis, (b) $5^{\circ}$ off the magnetic hard axis, (c) magnetic hard axis. The insets are close-ups of the regions between $-5 \mathrm{mT}$ and $+5 \mathrm{mT}$. 


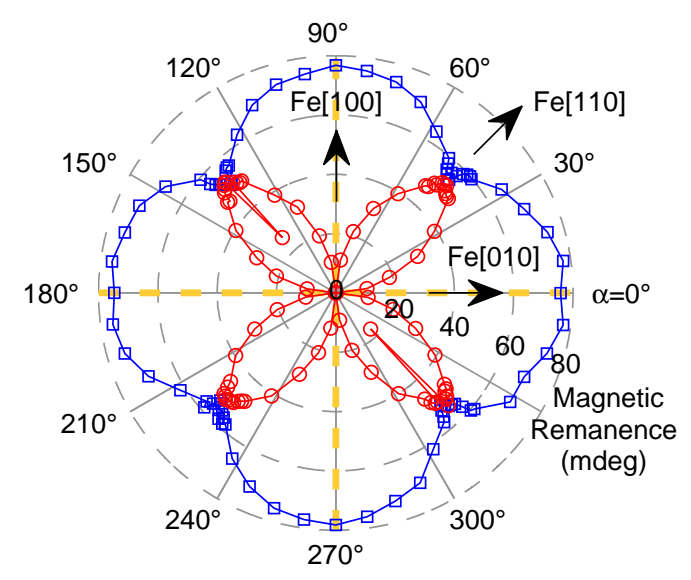

FIG. 7. Magnetic remanence of the $M_{1}$ component (circles) and of the $M_{2}$ component (squares) of the magnetization vector plotted against the azimuthal sample angle $\alpha$. The yellow dashed lines denote the magnetic easy axes. Please, note that the third crystallographic direction $\mathrm{Fe}(001)$ points away from the observer. 
(a)

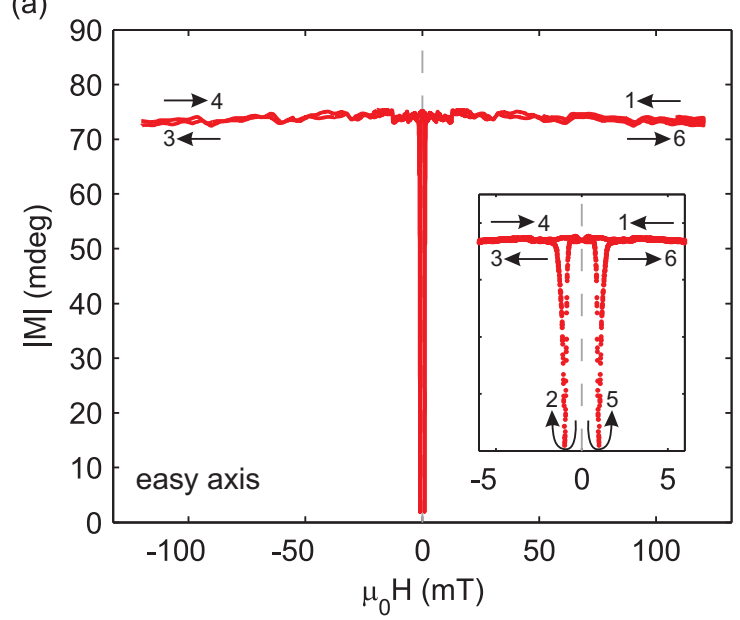

b)

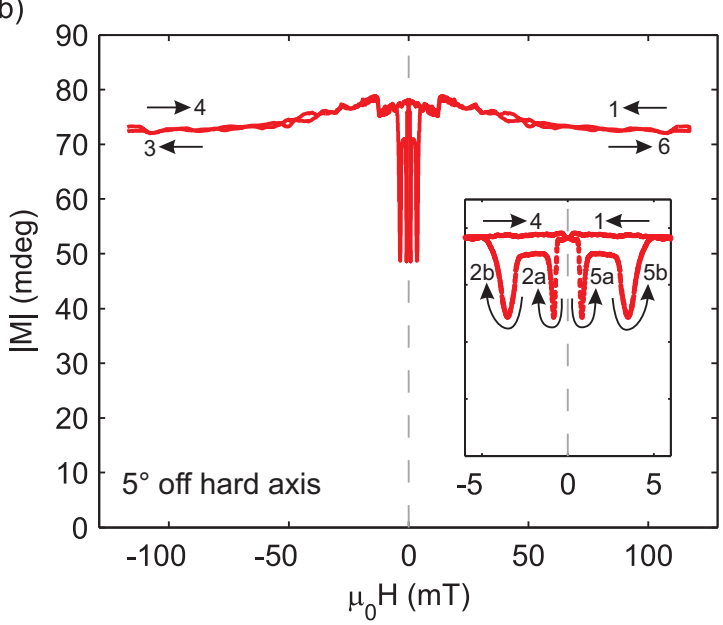

(c)

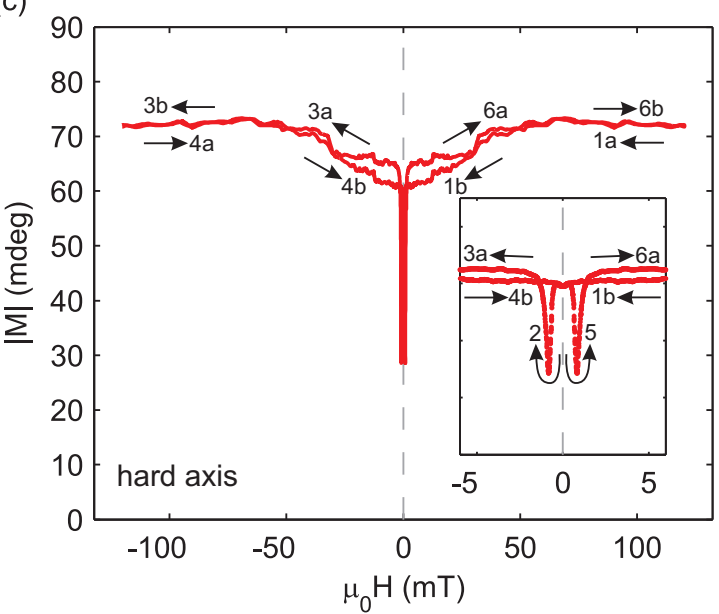

FIG. 8. Magnitude of the magnetization vector $|\vec{M}|$ during the reversal process calculated from the magnetization curves for $M_{1}$ and $M_{2}$ shown in Fig. 6 for (a) magnetic easy axis, (b) $5^{\circ}$ off the magnetic hard axis, (c) magnetic hard axis. The numbers in the curves denote the sequence of the reversal process. The insets are close-ups of the regions between $-5 \mathrm{mT}$ and $+5 \mathrm{mT}$. 
(a)

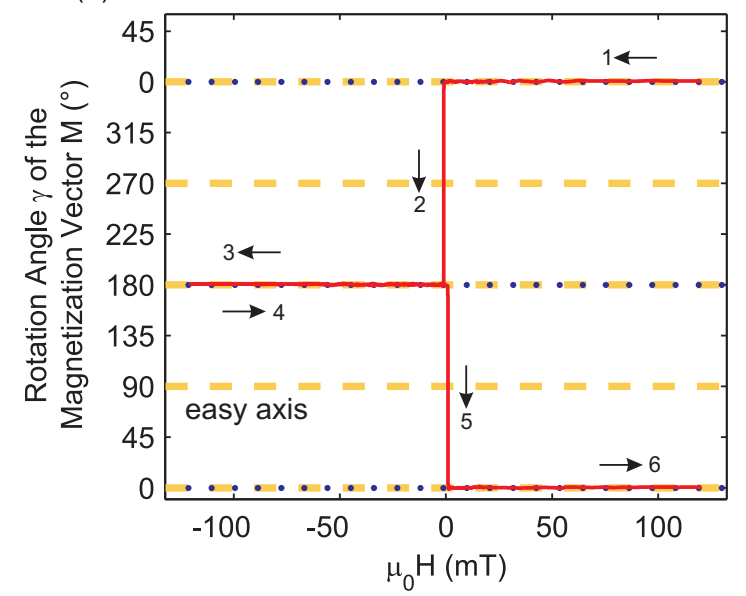

(b)

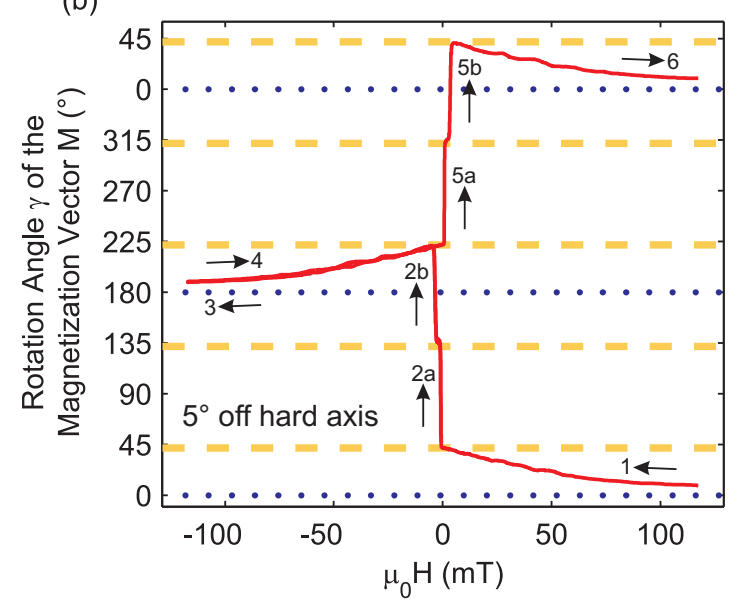

(c)

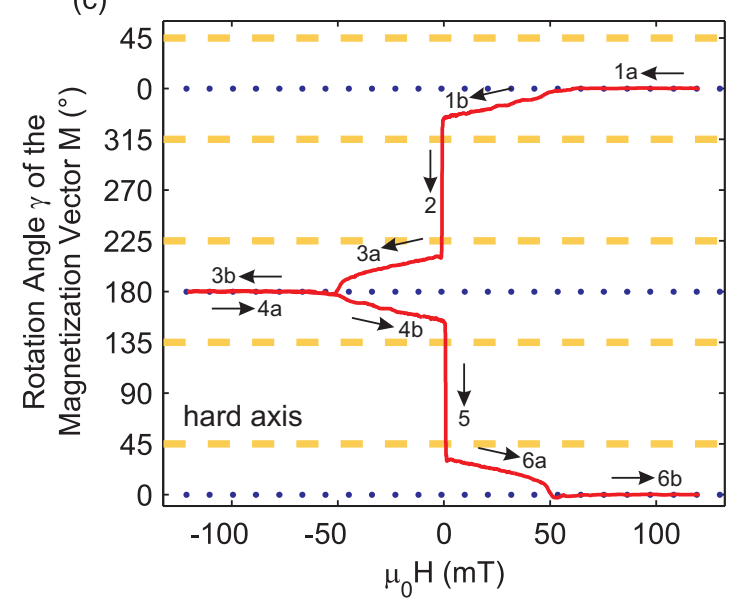

FIG. 9. Rotation angle $\gamma$ of the magnetization vector $\vec{M}$ during the reversal process calculated from the magnetization curves for $M_{1}$ and $M_{2}$ shown in Fig. 6 for (a) magnetic easy axis, (b) $5^{\circ}$ off the magnetic hard axis, (c) magnetic hard axis. The external magnetic field is aligned in $\gamma=0^{\circ}$ and $\gamma=180^{\circ}$ direction, respectively (blue dots). The yellow dashed lines denote the magnetic easy axes of the Fe film. The numbers in the curves denote the sequence of the reversal process. 

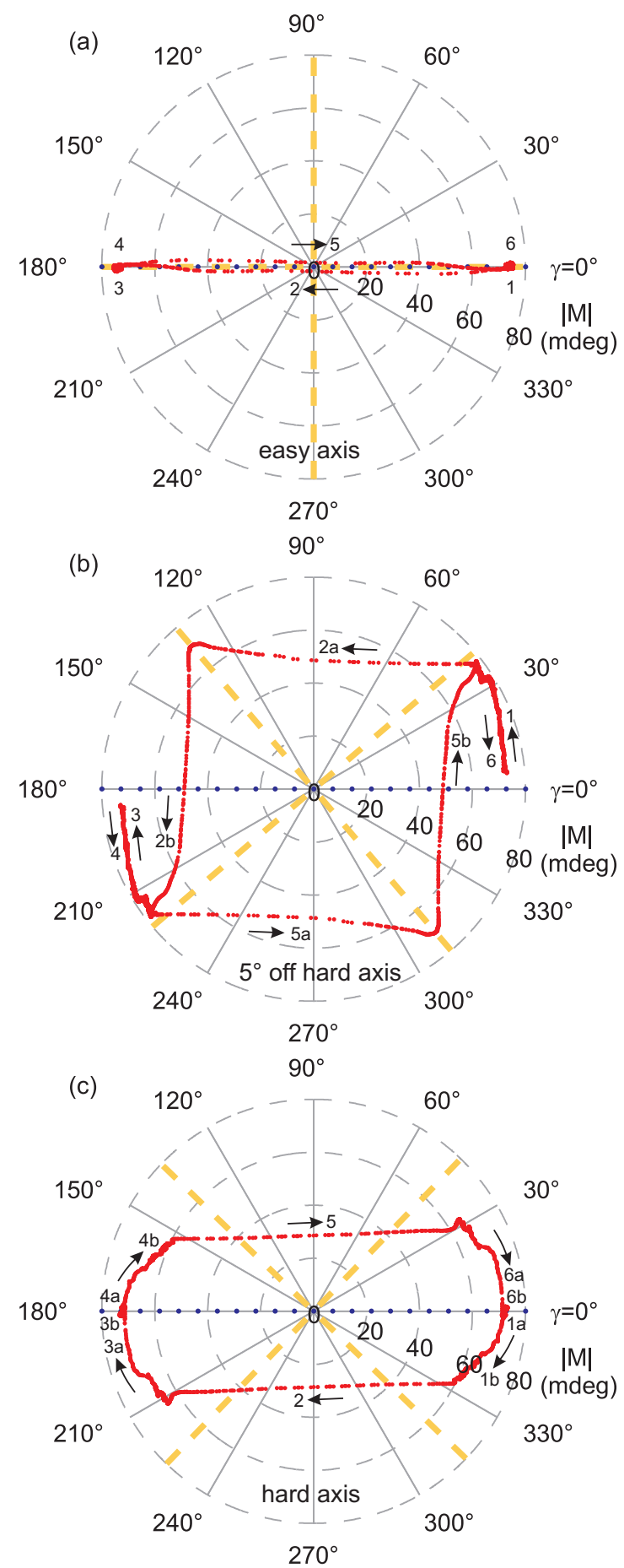

FIG. 10. Reversal process of the magnetization vector for (a) magnetic easy axis, (b) $5^{\circ}$ off the magnetic hard axis, (c) magnetic hard axis. The magnitude of the magnetization vector $|\vec{M}|$ in Fig. 8 is plotted against the rotation angle $\gamma$ of the magnetization vector $\vec{M}$ in Fig. 9. The external magnetic field is aligned in $\gamma=0^{\circ}$ and $\gamma=180^{\circ}$ direction, respectively (blue dots). The yellow dashed lines denote the magnetic easy axes of the Fe film. The numbers in the curves denote the sequence of the reversal process. 

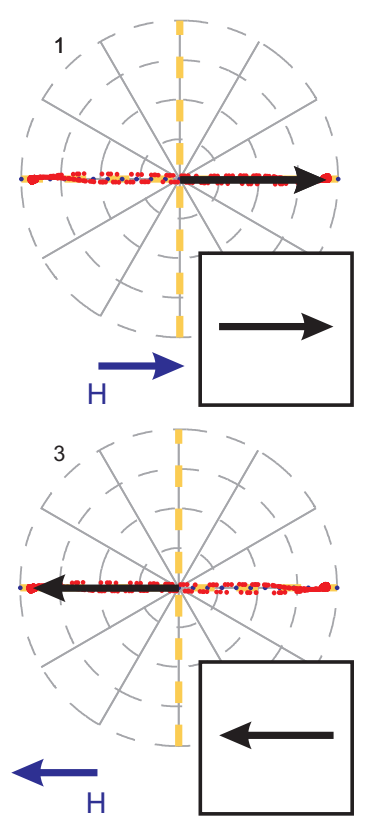

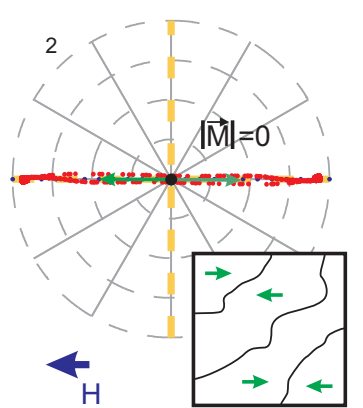

easy axis

FIG. 11. Analysis of the reversal process of the magnetization vector with an external magnetic field parallel to the magnetic easy axis. Lower left corner: Strength of the external magnetic field. Lower right corner: Possible domain structure. The indication of sequence numbers and lines equate the labeling in Fig. 10(a). 

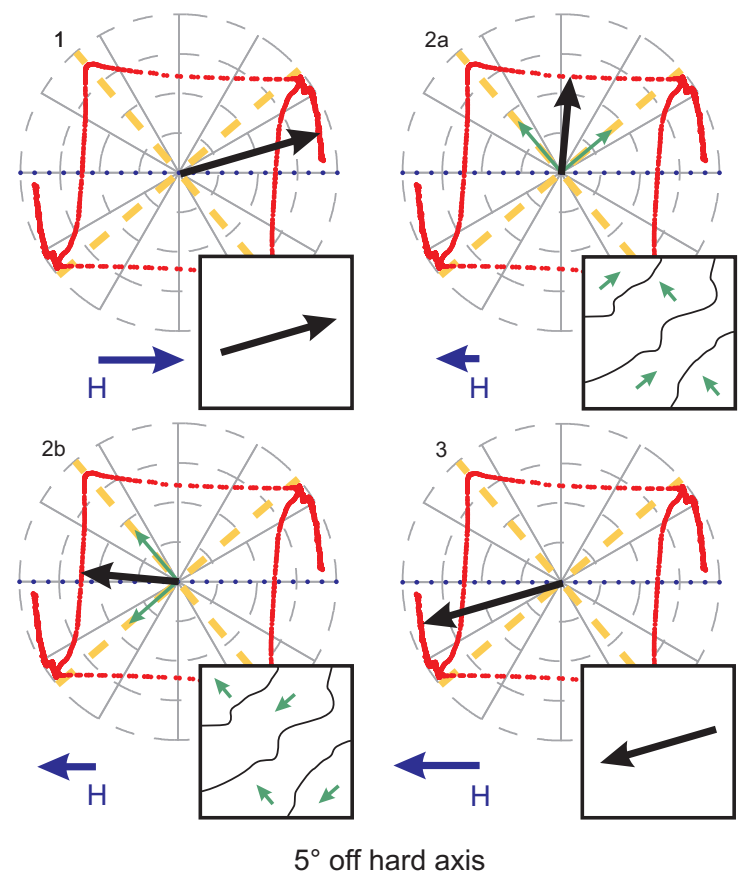

FIG. 12. Analysis of the reversal process of the magnetization vector with an external magnetic field $5^{\circ}$ off the magnetic hard axis. Lower left corner: Strength of the external magnetic field. Lower right corner: Possible domain structure. The indication of sequence numbers and lines equate the labeling in Fig. 10(b). 

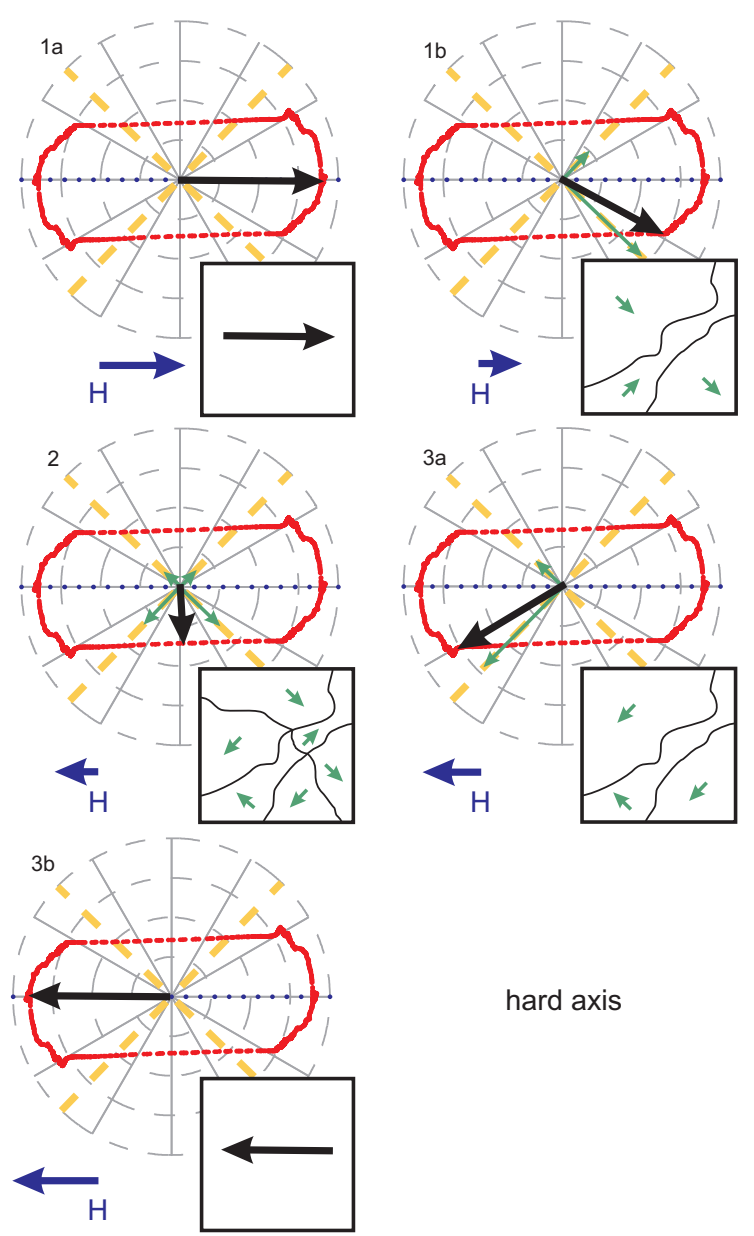

hard axis

FIG. 13. Analysis of the reversal process of the magnetization vector with an external magnetic field parallel to the magnetic hard axis. Lower left corner: Strength of the external magnetic field. Lower right corner: Possible domain structure. The indication of sequence numbers and lines equate the labeling in Fig. 10(c). 